\title{
Optically Pumped Mixing in Photonically Integrated Uni-Travelling Carrier Photodiode
}

\author{
Ahmad W. Mohammad ${ }^{1}$, Andrzej Jankowski², Frederic van Dijk ${ }^{2}$, and Cyril C. Renaud ${ }^{1}$ \\ ${ }^{1}$ Department of Electronic and Electrical Engineering, University College London, Torrington Place, London, \\ WC1E 7JE, UK \\ ${ }^{2}$ III-V Lab, a joint Laboratory of "Nokia Bell Labs", "Thales Research \& Technology" and "CEA-LETI", \\ Palaiseau, France
}

\begin{abstract}
We report the first demonstration of optically pumped mixing using a monolithically integrated photonic chip. On that chip, uni-traveling carrier photodiodes (UTC-PDs) were monolithically integrated with two lasers to generate the optical heterodyne that will drive the optically pumped mixing. The two DFB lasers wavelength spacing was tuneable from $70.5 \mathrm{GHz}$ to 92.4 GHz. When an RF signal at $70 \mathrm{GHz}$ was supplied to the UTCPD with the optimum voltage bias, the UTC-PD successfully downconverted the RF signal to an intermediate frequency (IF) that was tuneable from $0.5 \mathrm{GHz}$ to $16.4 \mathrm{GHz}$. These results demonstrate the potential of this photonic integrated circuit in spectroscopy, sensing and as millimeter wave wireless receivers.
\end{abstract}

\section{INTRODUCTION}

$\mathrm{T}$ HE characteristics of the millimeter waves (MMWs) have attracted the interest of many fields. For instance, the short wavelength of the MMWs increases distance resolution in radar systems. Also, the abundance of spectrum in the MMW range $(30 \mathrm{GHz}$ - $300 \mathrm{GHz})$ allows for high speed communications, while the high propagation loss makes it more secure. Moreover, MMWs have found interesting applications in imaging, sensing, and medicine [1].

Previously, optically pumped mixing (OPM) has been demonstrated at $100 \mathrm{GHz}$ using a single non-integrated unitraveling carrier photodiode (UTC-PD) [2, 3].

In this paper, we report OPM using a UTC-PD that is monolithically integrated with two lasers and semiconductor optical amplifiers (SOAs). The lasers generated optical tones with tuneable spacing between $70.5 \mathrm{GHz}$ and $92.4 \mathrm{GHz}$. When an RF signal at $70 \mathrm{GHz}$ was supplied to the UTC-PD with the optimized voltage bias for mixing, the UTC-PD downconverted the RF signal to an intermediate frequency (IF) that was tuneable from $0.5 \mathrm{GHz}$ to $16.4 \mathrm{GHz}$.

\section{DEVICE DESCRIPTION}

As shown in Fig. 1, the monolithically integrated chip used in this work [4] incorporates two UTC-PDs, two distributed feedback (DFB) lasers to provide the optical heterodyne, several SOAs to amplify the optical signals, multimode interference (MMI) couplers and electro-absorption modulators (EAMs). Moreover, this chip has an optical monitoring output.

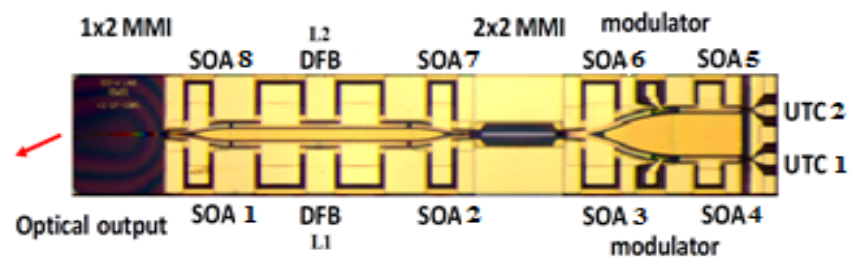

Fig. 1. Picture of the photonic integrated circuit.

\section{HETERODYNING EXPERIMENT}

In order to assess the performance of the PIC as an optical heterodyne MMW generator, we conducted a heterodyning experiment (Fig. 2), by which the lasers and SOAs were biased using a multi-contact DC probe, and the generated electrical heterodyne signal $(\Delta \mathrm{F})$ was extracted using a coplanar probe from UTC 1, which is shown in Fig. 1. Consequently, SOA5 and SOA6 were disconnected as they are in the optical path of UTC 2. Also, SOA1 and SOA8, which are used to amplify the optical signal for monitoring purposes, were disconnected to minimize reflections. The total current supplied to the other SOAs was fixed at $467 \mathrm{~mA}$. A bias Tee was used to apply the voltage bias to the UTC$\mathrm{PD}$, which was fixed at $-2 \mathrm{~V}$.

By monitoring the optical output of the photonic integrated circuit (PIC) we observed that the DFB lasers' threshold currents were $40 \mathrm{~mA}$ for $\mathrm{L} 1$ and $54 \mathrm{~mA}$ for L2. The lasers bias currents were gradually increased (up to $113 \mathrm{~mA}$ for L1 and $100 \mathrm{~mA}$ for L2) causing their operating wavelengths spacing to change. The wavelength tuning was observed by monitoring both the optical spectra and the generated electrical heterodyne. Wide tuneability from $70.5 \mathrm{GHz}$ to $92.4 \mathrm{GHz}$ was achieved, as shown in Fig. 3. The electrical spectra of the generated electrical heterodyne signals were measured, as shown in Fig. 4, using an electrical spectrum analyzer (ESA) which has a $75 \mathrm{GHz}$ to $110 \mathrm{GHz}$ mixer at its input.

Further, photocurrent variation between $6.5 \mathrm{~mA}$ and 7.2 $\mathrm{mA}$ was observed. This is due to the large variation in the lasers bias currents, as shown in Fig. 3, which caused the power of the optical signal at the input of the photodiode to vary.

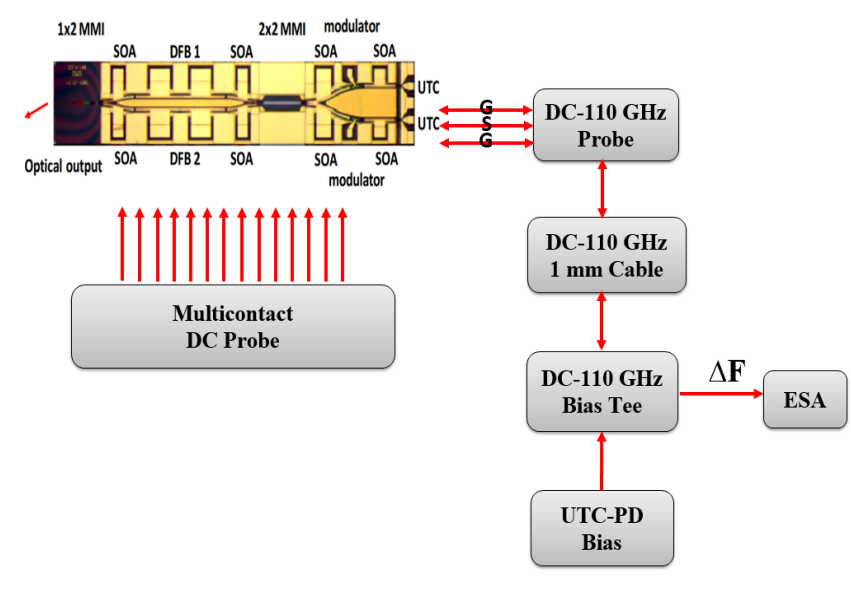

Fig. 2. Optical heterodyning experiment. 


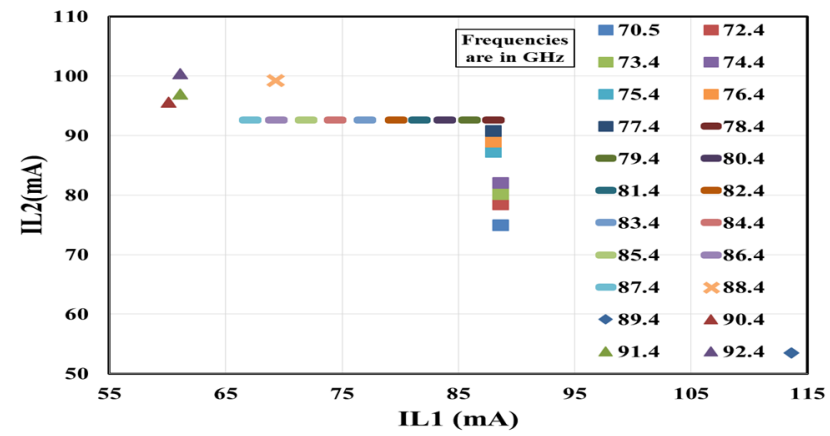

Fig. 3. Wide tuneability of the electrical heterodyne.

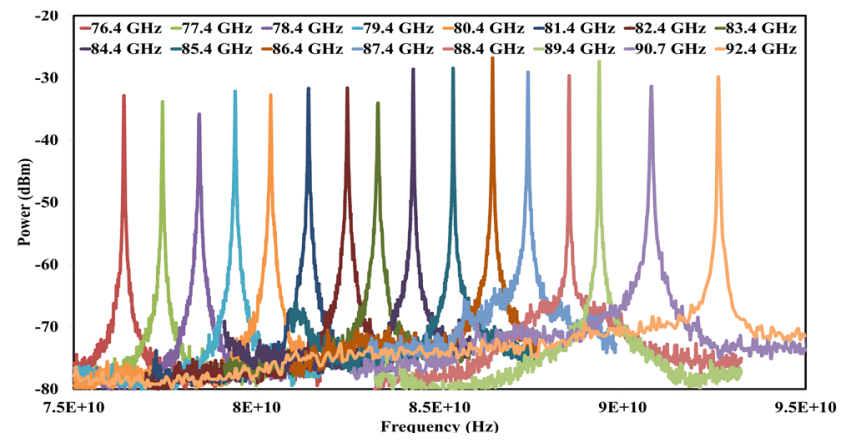

Fig. 4. Spectra of the electrical heterodyne $(\mathrm{RBW}=300 \mathrm{kHz})$.

\section{OPTICALLY PUMPED MIXING EXPERIMENT}

The block diagram of our OPM experiment is shown in Fig. 5. In OPM, the UTC-PD is injected with two optical tones to generate the electrical heterodyne signal at $\Delta \mathrm{F}$. When an $\mathrm{RF}$ signal is supplied to the UTC-PD with a voltage bias, the UTCPD generates a replica of the RF signal at IF. The power of the generated IF is maximized as the optimum voltage is supplied.

The received RF signal was generated by a signal synthesizer at $70 \mathrm{GHz}$, coupled, using a bias Tee, with a UTC-PD voltage bias that is optimum for mixing, and sent to the UTC-PD via a coplanar probe. The optimum biasing voltage was found by sweeping the applied voltage bias while monitoring the power of the IF signal. The optimum voltage bias varied between $-1 \mathrm{~V}$ and $-1.4 \mathrm{~V}$ across the IF range, while the photocurrent varied between $3.2 \mathrm{~mA}$ and $4.5 \mathrm{~mA}$. These variations are attributed to the change in the optical signal power at the UTC-PD input as the lasers bias currents were tuned. A power splitter was used to allow for the simultaneous supply of the RF to the UTC-PD and the extraction of IF from the UTC-PD. After considering the typical losses of the components in the RF path, the RF signal received at the UTC-PD was estimated at $-7 \mathrm{dBm}$.

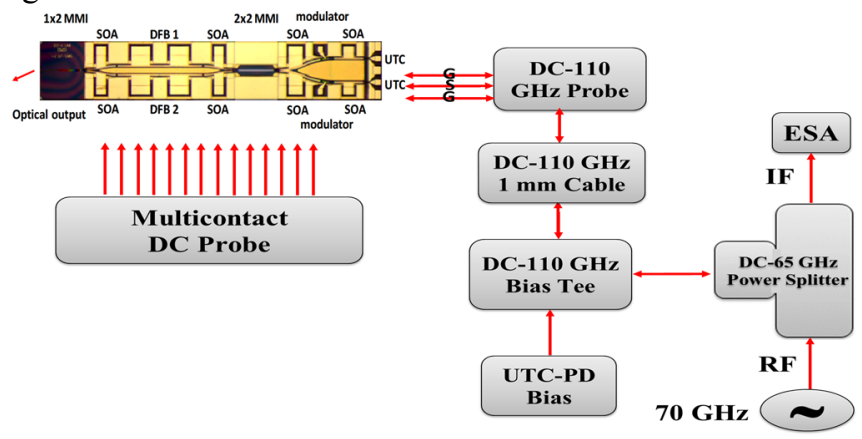

Fig. 5. Block diagram of the optically pumped mixing experiment.

\section{OPM EXPERIMENT RESULTS}

Fig. 6 shows the electrical spectra after down-conversion in the range from $0.5 \mathrm{GHz}$ to $16.4 \mathrm{GHz}$, while Fig. 7 shows the conversion loss (CL) performance across the IF range. Here, CL is defined as the ratio between the estimated power of the incoming RF signal to the UTC-PD, and the generated IF signal power, at a UTC-PD bias voltage that is optimum for mixing. Fig. 7 shows a flat $\mathrm{CL}$ across the IF range except at $0.5 \mathrm{GHz}$ due to impedance mismatching at this frequency [2]. The CL values presented here are higher than the values reported in $[2,3]$ because the UTC-PDs on this PIC are optimized for MMW emission rather than mixing. Results around $10 \mathrm{GHz}$ are not shown due to lasers relaxation oscillation at this frequency, which interfered with the down-converted IF.

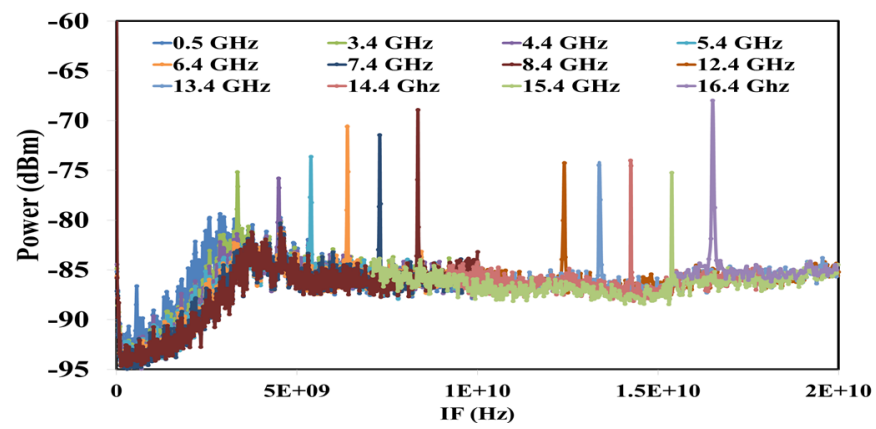

Fig. 6. Wide tuneability of IF $(\mathrm{RBW}=1 \mathrm{MHz})$.

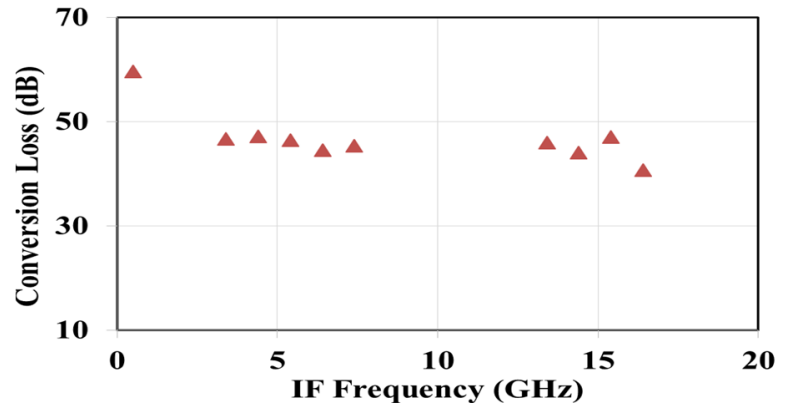

Fig. 7. Conversion loss across IF range.

\section{CONCLUSION}

We demonstrated optically pumped mixing on a PIC, with a relatively flat conversion loss across a wide frequency range from $3.5 \mathrm{GHz}$ to $16.4 \mathrm{GHz}$. Considering that a similar PIC was used as MMW emitter [4] and the potential bandwidth for down-conversion, such a PIC would offer a compact MMW transceiver for a range of applications.

\section{REFERENCES}

[1] Technology Report, "A growing number of applications boosts mm-wave technology," High Frequency Electronics, vol. 5, pp. 52-53, May, 2006.

[2] E. Rouvalis, M. J. Fice, C. C. Renaud, and A. J. Seeds, "Millimeter-wave optoelectronic mixers based on uni-traveling carrier photodiodes," IEEE Trans. Microw. Theory Tech., vol. 60, pp. 686-691, Jan, 2012.

[3] Ahmad Mohammad, Haymen Shams, Katarzyna Balakier, Chris Graham, Michele Natrella, Alwyn J. Seeds, and Cyril C. Renaud, "5 Gbps wireless transmission link with an optically pumped uni-traveling carrier photodiode mixer at the receiver," Opt. Express, vol. 26, pp. 2884-2890, Jan, 2018.

[4] F. van Dijk, G. Kervella, M. Lamponi, M. Chtioui, F. Lelarge, C. Renaud, G. Carpintero, and M. Alouini, "Wireless data transmission and frequency stabilization with a millimeter-wave photonic integrated circuit," Proc. IEEE Topical Meeting Microw. Photon., pp. 1-4, Oct., 2015. 